This item was submitted to Loughborough's Research Repository by the author.

Items in Figshare are protected by copyright, with all rights reserved, unless otherwise indicated.

\title{
Continuous nonsingular terminal sliding mode control for nonlinear systems subject to mismatched terms
}

\author{
PLEASE CITE THE PUBLISHED VERSION
}

https://doi.org/10.1002/asjc.2486

\section{PUBLISHER}

Wiley

\section{VERSION}

AM (Accepted Manuscript)

\section{PUBLISHER STATEMENT}

This is the peer reviewed version of the following article: Zhang, L, Yang, J. Continuous nonsingular terminal sliding mode control for nonlinear systems subject to mismatched terms. Asian J Control. 24 (2022), 885894, which has been published in final form at https://doi.org/10.1002/asjc.2486. This article may be used for non-commercial purposes in accordance with Wiley Terms and Conditions for Use of Self-Archived Versions. This article may not be enhanced, enriched or otherwise transformed into a derivative work, without express permission from Wiley or by statutory rights under applicable legislation. Copyright notices must not be removed, obscured or modified. The article must be linked to Wiley's version of record on Wiley Online Library and any embedding, framing or otherwise making available the article or pages thereof by third parties from platforms, services and websites other than Wiley Online Library must be prohibited.

\section{LICENCE}

CC BY-NC-ND 4.0

\section{REPOSITORY RECORD}

Zhang, Lu, and Jun Yang. 2020. "Continuous Nonsingular Terminal Sliding Mode Control for Nonlinear Systems Subject to Mismatched Terms". Loughborough University. https://hdl.handle.net/2134/16811329.v1. 


\title{
Continuous Nonsingular Terminal Sliding Mode Control for Nonlinear Systems Subject to Mismatched Terms ${ }^{\dagger}$
}

\author{
Lu Zhang $^{1}$, Jun Yang ${ }^{2 \ddagger}$ \\ ${ }^{1}$ School of Automation, Southeast University, Key Laboratory of Measurement and Control of CSE, \\ Ministry of Education, Nanjing 210096, China \\ ${ }^{2}$ Department of Aeronautical and Automotive Engineering, Loughborough University, \\ Loughborough LE11 3TU, UK
}

\begin{abstract}
This paper proposes a continuous nonsingular terminal sliding mode (NTSM) control approach for nonlinear systems subject to mismatched terms in order to achieve finite time exact tracking and disturbance rejection. The controller is constructed using a composite method that utilizes a power integrator and combines the output regulation theory, disturbance observation technique, feedback domination and sliding mode control technique. The performance analysis demonstrates that the proposed continuous NTSM controller can drive the system output to the desired reference signal in a finite time in the presence of mismatched time-varying disturbances and nonsmoothed nonlinearities in each channel. The finite time Lyapunov theory is utilized to ensure finite-time convergence of the closed-loop system. The simulation results validate the effectiveness of the proposed continuous NTSM controller.
\end{abstract}

Key words Continuous nonsingular terminal sliding mode control, finite-time exact tracking, finite-time disturbance observer, Lyapunov function.

\footnotetext{
${ }^{\dagger}$ The work was supported in part by the National Natural Science Foundation of China under Grant 61973080 and Grant 61973081.

‡To whom all correspondence should be sent. E-mail: j.yang3@lboro.ac.uk.
} 


\section{INTRODUCTION}

Sliding mode control (SMC) is proved to be effective in dealing with internal parameter variations and external disturbances with low computational complexity $[1,2]$. The conventional SMC adopts the linear sliding manifold and the system states reach their equilibrium points asymptotically. In recent years, the terminal sliding mode (TSM) [3] is proposed, which can drive the system states to their equilibrium points in a finite-time by designing a nonlinear sliding manifold. However, the TSM method has the singular problem [3]. In order to overcome this disadvantage, the nonsingular terminal sliding mode (NTSM) has been proposed in [4]. The NTSM approaches can provide finite-time stability, fast dynamic response, low steady-state errors, more degrees of freedom for parameter choice of the controller and avoid the singular problem. Due to these advantages, the NTSM controllers are widely used in various applications, such as electronic throttle systems [5], autonomous underwater vehicles [6], dual-motor driving servo systems [7], robot manipulators [8] and so on.

In practice, with the development of industrial systems, there are not only matched disturbances/uncertainties in the systems, but also mismatched disturbances and nonlinearities [9, 10]. For example, the load torque is the mismatched disturbance in permanent magnet asynchronous motor system [11]. In [12], a nonlinear sliding mode controller based on linear matrix inequality has been proposed to handle the mismatched disturbances. In [13], a novel sliding surface has been proposed to simultaneously achieve low overshoot and short settling time for the system with mis- matched disturbances. However, the controllers proposed in [12] and [13] cannot drive the system states to the desired values in a finite-time. Even though the NTSM controllers have several advantages, they cannot effectively cope with the mismatched disturbances/uncertainties, which is the same problem as in the traditional SMC [1], therefore, designing a NTSM controller to counteract the mismatched disturbances/uncertainties and nonlinearities is challenging and significant.

To date, several methods have been proposed to solve the mismatched problem in NTSM by combining different robust approaches. In [14], a continuous nonsingular terminal sliding mode controller has been proposed for small-scale unmanned helicopter with mismatched disturbances. In [15], an anti-disturbance control method based on the non-singular terminal sliding mode theory has been proposed for leader-follower multiagent system with mismatched disturbances. In [16], the NTSM control has been used to solve the load disturbances in DC-DC buck converters. Moreover, a non-singular terminal sliding mode controller for flexible air-breathing hypersonic vehicle system has been proposed in [17]. In general, the existing methods can be divided into two categories. The first one is based on the principle to remove all the mismatched disturbances in the control channel through coordinate transformation $[18,19]$, and the disturbances can be suppressed by conventional NTSM controller [20, 21]. In [22], an adaptive fuzzy TSM controller has been proposed, which requires the mismatched disturbances to be $H_{2}$ norm-bounded. A full-order TSM controller has been proposed in [23] for MIMO systems subject to mismatched uncertainties, but the system output cannot converge to zero in a finite- 
time. In [24], an integral TSM controller has been proposed for hypersonic vehicles system with mismatched disturbances, but as is well known, the integral action will always bring adverse effects to control systems. It should be pointed out that the above-mentioned approaches will sacrifice the performance of the closed-loop system. The second category utilizes the disturbance observation technique [9] to online estimate the mismatched disturbances and compensate them in the controller. In [25], a continuous NTSM control method based on the finite-time disturbance observer (FTDO) [26] has been proposed to drive the system states to the set-point in a finite-time in the presence of mismatched disturbance. In [27], a disturbance observer based NTSM control method has been proposed for spacecraft system. Nevertheless, if nonlinearities exist in each channel of the system, the above-mentioned disturbance observer-based SMC methods will not be effective.

This paper aims to solve the finite-time exac$\mathrm{t}$ tracking and disturbance rejection problem for nonlinear systems with mismatched disturbances. A novel continuous NTSM control strategy is proposed based on the basic idea of output regulation theory $[28,29,30]$. The proposed control approach uses a power integrator and combines disturbance observation technique, feedback domination and sliding mode technique. Firstly, the paper provides a continuous NTSM control algorithm for handling the nonlinear systems with matched and mismatched disturbances as well as matched and mismatched nonsmoothed nonlinearities. Secondly, it is shown by finite-time Lyapunov theory that the proposed sliding mode controller achieves the finite-time exact tracking for the closed-loop system under mismatched time- varying disturbances. Finally, the proposed sliding mode controller is continuous, which largely reduces the adverse influence caused by the discontinuous switching of the control law.

\section{Preliminaries}

\section{$2.1 \quad$ Useful lemmas}

Firstly, some useful lemmas are presented that play important roles in the analysis. Define a $\mathcal{C}^{0}$ function as $\lfloor x\rceil^{\alpha}=|x|^{\alpha} \operatorname{sgn}(x)$.

Lemma 1 [31] For $x \in \mathbb{R}, y \in \mathbb{R}, p_{1}>0$ and $0<p_{2} \leq 1$, there is $\left|\lfloor x\rceil^{p_{1} p_{2}}-\lfloor y\rceil^{p_{1} p_{2}}\right| \leq$ $2^{1-p_{2}}\left|\lfloor x\rceil^{p_{1}}-\lfloor y\rceil^{p_{1}}\right|^{p_{2}}$.

Lemma 2 [31] For $x \in \mathbb{R}, y \in \mathbb{R}, c>0, d>0$ and any real-valued function $\gamma(x, y)>0$, the following inequality holds: $|x|^{c}|y|^{d} \leq \frac{c}{c+d} \gamma(x, y)|x|^{c+d}+$ $\frac{d}{c+d} \gamma^{-\frac{c}{d}(c+d)}(x, y)|y|^{c+d}$.

Lemma 3 [32] For $x_{i} \in \mathbb{R}, i=1, \cdots, n$ and $0<p \leq 1$, there is $\left(\left|x_{1}\right|+\cdots+\left|x_{n}\right|\right)^{p} \leq\left|x_{1}\right|^{p}+$ $\cdots+\left|x_{n}\right|^{p}$.

\subsection{Problem formulation}

Consider the following nonlinear system:

$$
\begin{aligned}
\dot{x}_{i}(t) & =x_{i+1}(t)+f_{i}\left(\bar{x}_{i}(t)\right)+\omega_{i}(t), \\
\dot{x}_{n}(t) & =u(t)+f_{n}\left(\bar{x}_{n}(t)\right)+\omega_{n}(t), \\
y(t) & =x_{1}(t),
\end{aligned}
$$

for $\bar{x}_{i}:=\left[x_{1}, \cdots, x_{i}\right]^{T}, i \in \mathbb{N}_{1: n-1}$ and $\mathbb{N}_{j: k}=$ $\{j, j+1, \cdots, k\}$ is a set of nonnegative integers. $x_{i} \in \mathbb{R}, u \in \mathbb{R}, y \in \mathbb{R}$ are the system state, the control input and the control output, respectively. $\omega_{i} \in \mathbb{D} \subset \mathbb{R}$ with $\mathbb{D}$ is a compact set that denotes the external disturbance. It is assumed that the 
external disturbance $\omega_{i}(t), i \in \mathbb{N}_{1: n}$ is unknown but its amplitude and derivatives are bounded by $\sup _{t>0}\left|\frac{d^{j} \omega_{i}(t)}{d t^{j}}\right| \leq \mathcal{F}_{\omega_{i}}^{+}$for $j \in \mathbb{N}_{0: n+1-i}$, where $\mathcal{F}_{\omega_{i}}^{+}$ is a positive constant. The state of $x_{i}$ and the nonlinear function $f_{i}\left(\bar{x}_{i}\right)$ are assumed to be available for control design, and $f_{i}\left(\bar{x}_{i}\right)$ is supposed to satisfy the following condition:

$$
\left|f_{i}\left(\bar{x}_{i}\right)-f_{i}\left(\bar{y}_{i}\right)\right| \leq \rho_{i}\left(\bar{x}_{i}, \bar{y}_{i}\right) \sum_{j=1}^{i}\left|x_{j}-y_{j}\right|^{\left(r_{i}+\tau\right) / r_{j}}
$$

for $i \in \mathbb{N}_{1: n}, r_{i+1}=r_{i}+\tau, \tau \in(-\infty, 0)$, with $r_{1}>0$ is a constant, and $\rho_{i}\left(\bar{x}_{i}, \bar{y}_{i}\right)>0$ is a $\mathcal{C}^{0}$ function.

Control Objective: This paper attempts to utilize the nonsingular terminal sliding mode control theory to address the finite-time exact tracking and disturbance rejection problem for a class of lower triangular nonlinear system (1), in which nonlinearities and time-varying disturbances exist in each channel. For system (1) satisfying (2), a recursive sliding manifold and continuous nonsingular terminal sliding mode controller are designed to ensure the finite-time exact tracking of the nonlinear system in the presence of matched and/or mismatched disturbances. Under the proposed controller, it is shown that there exists a finite-time $T$ such that $y(t)=r(t)$ for $t \geq T$.

\subsection{Motivations}

To begin with, the basic principle of TSM control is briefly introduced [3]. Consider the following second-order uncertain nonlinear system:

$$
\begin{aligned}
& \dot{x}_{1}=x_{2}, \\
& \dot{x}_{2}=f\left(x_{1}, x_{2}\right)+g\left(x_{1}, x_{2}, d\right)+u,
\end{aligned}
$$

where $f\left(x_{1}, x_{2}\right)$ is a smooth nonlinear function, and $g\left(x_{1}, x_{2}, d\right)$ represents the uncertainties and disturbances satisfying $\left|g\left(x_{1}, x_{2}, d\right)\right|<g^{*}$ with $g^{*}>0 . u$ is the control input.

The traditional first-order terminal sliding manifold is designed as $s=x_{2}+\beta x_{1}^{q / p}$, where $\beta>0$ is a constant to be designed, $p, q$ are positive integers satisfying $p>q$. The sufficient condition for the existence of TSM is $\frac{1}{2} \frac{d}{d t} s^{2} \leq-k|s|$, where $k>0$ is a constant. For system (3), the TSM controller is designed as

$u=-\left[f\left(x_{1}, x_{2}\right)+\beta q / p x_{1}^{q / p-1} x_{2}+\left(g^{*}+k\right) \operatorname{sgn}(s)\right]$.

It is observed that if $s(0) \neq 0$, the system states will reach the sliding manifold $s=0$ in a finite-time $t_{r}$ with $t_{r} \leq \frac{|s(0)|}{\eta}$. When the sliding manifold is reached, the system dynamics are given by $x_{2}+\beta x_{1}^{q / p}=\dot{x}_{1}+\beta x_{1}^{q / p}=0$. Therefore, the system states $x_{1}$ and $x_{2}$ converge to zero in a finite-time.

Since the controller (4) contains the term $x_{1}^{q / p-1} x_{2}$, it may cause a singularity to occur if $x_{2} \neq 0$ when $x_{1}=0$. The TSM controller (4) cannot ensure a bounded control signal for the case of $x_{2} \neq 0$ when $x_{1}=0$ before the system states reach the sliding manifold. Moreover, the singularity problem may also occur after the sliding manifold is reached due to the uncertain factors in the closed-loop system. It can be ensured that the system states will always remain in the sliding mode especially near the equilibrium point and the case of $x_{2} \neq 0$ while $x_{1}=0$ may occur from time to time [4].

\section{Main results}

\subsection{Steady-state estimations}

A set of FTDO [26] are designed as follows to estimate the disturbances and their derivatives in 
system (1):

$$
\begin{aligned}
& \dot{z}_{0}^{i}=v_{o}^{i}+x_{i+1}+f_{i}\left(\bar{x}_{i}(t)\right), \\
& \dot{z}_{1}^{i}=v_{1}^{i}, \ldots, \dot{z}_{n-i+1}^{i}=v_{n-i+1}^{i},
\end{aligned}
$$

for $i=1, \ldots, n$, with definitions $x_{n+1}=u$, $v_{o}^{i}=-\lambda_{0}^{i} L_{i}^{\frac{1}{n-i+2}}\left\lfloor z_{0}^{i}-x_{i}\right\rceil^{\frac{n-i+1}{n-i+2}}+z_{1}^{i}$, and $v_{j}^{i}=$ $-\lambda_{j}^{i} L_{i}^{\frac{1}{n-i+2-j}}\left\lfloor z_{j}^{i}-v_{j-1}^{i}\right\rceil^{\frac{n-i+1-j}{n-i+2-j}}+z_{j+1}^{i}$ for $i \in$ $\mathbb{N}_{1: n}, j \in \mathbb{N}_{0: n-i+1} . \quad \lambda_{j}^{i}>0$ and $L_{i}>0$ is the observer gains to be designed. The estimations of $x_{i}$ and $\omega_{i}^{(k-1)}$ are given by $\hat{x}_{i}=z_{0}^{i}$ and $\hat{\omega}_{i}^{(k-1)}=z_{k}^{i}$, for $i \in \mathbb{N}_{1: n}, k \in \mathbb{N}_{1: n+1-i}$. According to [26], if the observer gain $L_{i}$ is selected to satisfy $L_{i}>\mathcal{F}_{\omega_{i}}^{+}$, then the above estimations converge to their true values in a finite-time $T_{1}$.

The steady-states of system (1) are determined by

$$
\begin{aligned}
x_{1 s}(t) & =r(t), \\
x_{(i+1) s}(t) & =\frac{d x_{i s}(t)}{d t}-f_{i}\left(\bar{x}_{i s}(t)\right)-\omega_{i}(t), \\
u_{s}(t) & =\frac{d x_{n s}(t)}{d t}-f_{n}\left(\bar{x}_{n s}(t)\right)-\omega_{n}(t),
\end{aligned}
$$

where $i \in \mathbb{N}_{2: n}, x_{i s}(t)$ is the steady-state of $x_{i}(t)$. By virtue of disturbance estimations, the steadystate estimations are obtained as follows:

$$
\begin{aligned}
\hat{x}_{1 s}(t) & =r(t), \\
\hat{x}_{(i+1) s}(t) & =\frac{d \hat{x}_{i s}(t)}{d t}-f_{i}\left(\overline{\hat{x}}_{i s}(t)\right)-z_{1}^{i}(t), \\
\hat{u}_{s}(t) & =\frac{d \hat{x}_{n s}(t)}{d t}-f_{n}\left(\overline{\hat{x}}_{n s}(t)\right)-z_{1}^{n}(t),
\end{aligned}
$$

where $\hat{x}_{1 s}, \hat{x}_{i s}, \hat{u}_{s}$ are estimations of $x_{1 s}, x_{i s}, u_{s}$ for $i \in \mathbb{N}_{2: n}$. Suppose that $\hat{x}_{i s} \in \mathcal{C}^{1}$ for $i \in \mathbb{N}_{1: n}$ and $\hat{u}_{s} \in \mathcal{C}^{0}$ with respect to their respective arguments for all $t \geq 0$.

Lemma 4 The disturbance observers (5) ensure that the steady-state estimations of system (1) converge to their real steady-states in a finitetime $T_{2}$, that is, $\hat{x}_{i s}=x_{i s}, \hat{u}_{s}=u_{s}$ for all $t \geq T_{2}$.
Proof: Since $\hat{x}_{i s} \in \mathcal{C}^{1}$ and $\hat{u}_{s} \in \mathcal{C}^{0}$, it can be derived from the boundedness of disturbances, references and disturbance estimations that $x_{i}(t) \in$ $\mathcal{L}_{\infty}, \dot{x}_{i}(t) \in \mathcal{L}_{\infty}, u_{s}(t) \in \mathcal{L}_{\infty}, \hat{x}_{i}(t) \in \mathcal{L}_{\infty}, \hat{\dot{x}}_{i}(t) \in$ $\mathcal{L}_{\infty}$, and $\hat{u}_{s}(t) \in \mathcal{L}_{\infty}$. Since for all $i \in \mathbb{N}_{1: n}$ and $k \in \mathbb{N}_{1: n+1-i}$ the convergence of the disturbance observer (5) reveals that $z_{k}^{i}=\omega_{i}^{(k-1)}$ for all $t>T_{1}$, we can draw the conclusion that $\hat{x}_{i s}=x_{i s}, \hat{u}_{s}=u_{s}$ for all $t>T_{2}$.

Remark 1 Combining system dynamics (1) with the finite-time disturbance observer (5), the estimation error dynamics are given by

$$
\begin{aligned}
\dot{e}_{0}^{i} & =-\lambda_{0}^{i} L_{i}^{\frac{1}{n-i+2}}\left\lfloor e_{0}^{i}\right\rceil^{\frac{n-i+1}{n-i+2}}+e_{1}^{i}, \\
\dot{e}_{j}^{i} & =-\lambda_{j}^{i} L_{i}^{\frac{1}{n-i+2-j}}\left\lfloor e_{j}^{i}-\dot{e}_{j-1}^{i}\right\rceil^{\frac{n-i+1-j}{n-i+2-j}}+e_{j+1}^{i}, \\
\dot{e}_{n-i+1}^{i} & \in-\lambda_{n-i+1}^{i} L_{i} \operatorname{sgn}\left(e_{n-i+1}-\dot{e}_{n-i}^{i}\right)+\left[-L_{i}, L_{i}\right],
\end{aligned}
$$

where the estimation errors are defined as $e_{0}^{i}=$ $z_{0}^{i}-x_{i}, e_{j}^{i}=z_{j}^{i}-\omega_{i}^{(j-1)}$. According to [2], the differential inclusion is homogeneous with homogeneity degree -1 weights $n-i-j+2, n-i-j+1, \cdots, 1$ of $e_{0}^{i}, e_{1}^{i}, \cdots, e_{n-i+1}^{i}$, respectively, and the estimation errors will converge to zero in a finite-time $T_{1}$. In [26], it has been proven that the finite-time $T_{1}$ is related to the initial conditions and the observer parameters. Furthermore, the value of $T_{1}$ can be arbitrary small if the observer gains are selected sufficiently large enough [26], and the value of $T_{2}$ depends on $T_{1}$ and system dynamics.

\subsection{Continuous NTSM controller de- sign}

Denote $\eta_{i}=x_{i}-\hat{x}_{i s}$. Let $\rho$ satisfy $\rho \geq \max _{i \in \mathbb{N}_{1: n}}\left\{r_{i}\right\}$. A new NTSM manifold is designed as

$$
s(t)=\eta_{n}(t)-\eta_{n}(0)+\int_{0}^{t} \lambda_{n}\left(\bar{\eta}_{n}\right)\lfloor\sigma(\tau)\rceil^{\frac{r_{n+1}}{\rho}} d \tau,
$$


where $\sigma(t)=\sum_{i=1}^{n} \lambda_{i}\left(\bar{\eta}_{n}\right)\left\lfloor\eta_{i}\right\rceil^{\frac{\rho}{r_{i}}}, \lambda_{i}\left(\bar{\eta}_{n}\right)=\prod_{l=i}^{n} \kappa_{i}^{\frac{\rho}{r_{l+1}}}\left(\bar{\eta}_{l}\right)$, and $\kappa_{i}\left(\bar{\eta}_{i}\right), i=\in \mathbb{N}_{1: n}$ are nonlinear functions with respect to $\bar{\eta}_{i}$.

With the sliding manifold (9), a recursive NTSM controller is designed as follows:

$$
u=-\left[u_{e q}(t)+u_{r}(t)\right],
$$

where the equivalent control law is constructed as

$$
u_{e q}=\hat{u}_{s}-\lambda_{n}\left(\bar{\eta}_{n}\right)\lfloor\sigma(t)\rceil^{\frac{r_{n+1}}{\rho}}-f_{n}\left(\bar{x}_{n}\right)+f_{n}\left(\overline{\hat{x}}_{n s}\right) \text {, }
$$

and the reaching law is designed as

$$
u_{r}=k_{1}\lfloor s\rceil^{\frac{1}{2}}+k_{2} \int_{0}^{t}\lfloor s(\tau)\rceil^{0} d \tau,
$$

with $k_{1}>0$ and $k_{2}>0$ are parameters to be determined.

\section{Performance and stability anal- ysis}

The performance and stability analysis is based on Lyapunov theory, which is elaborated in this section.

Design the following dynamic compensators:

$$
\begin{aligned}
& \eta_{1}^{*}=0, \xi_{1}=\left\lfloor\left.\eta_{1}\right|^{\frac{\rho}{r_{1}}}\right. \\
& \eta_{i}^{*}=-\lambda_{i-1}\left(\bar{\eta}_{i-1}\right)\left\lfloor\xi_{i-1}\right\rfloor^{\frac{r_{i}}{\rho}} \\
& \xi_{i}=\left\lfloor\eta_{i}\right\rceil^{\frac{\rho}{r_{i}}}-\left\lfloor\eta_{i}^{*}\right\rceil^{\frac{\rho}{r_{i}}}
\end{aligned}
$$

for $i \in \mathbb{N}_{2: n}, \mu \geq \max _{i \in \mathbb{N}_{1: n}}\left\{r_{i+1}, \rho\right\}$. Next, a positive definite Lyapunov function is designed as

$$
V_{i}\left(\bar{\eta}_{i}\right)=\sum_{k=1}^{i} W_{k}\left(\bar{\eta}_{k}\right),
$$

with $W_{k}\left(\bar{\eta}_{k}\right)=\int_{\eta_{i}^{*}}^{\eta_{i}}\left\lfloor\lfloor q\rceil^{\frac{\rho}{r_{i}}}-\left\lfloor\eta_{i}^{*}\right\rceil^{\frac{\rho}{r_{i}}}\right\rfloor^{\frac{2 \mu-r_{i+1}}{\rho}} d q$.

Using the lemmas given in Section 2 and the Lyapunov function (14), the main result of this paper can be obtained, which is summarized by the following theorem.
Theorem 1 For the $n$ - th order nonlinear system (1) with mismatched terms, the finite-time exact tracking for the system output can be guaranteed using the finite-time disturbance observer based NTSM controller (5), (9)-(12), i.e. $y(t) \rightarrow$ $r(t)$ as $t \rightarrow T$ with $T$ is a finite-time.

Proof: Taking the time-derivative of $\eta_{i}$, one obtains

$$
\begin{aligned}
\dot{\eta}_{i} & =\eta_{i+1}+f_{i}\left(\bar{x}_{i}\right)-f_{i}\left(\overline{\hat{x}}_{i s}\right)+\varepsilon_{i}(t), i \in \mathbb{N}_{1: n-1}, \\
\dot{\eta}_{n} & =u-\hat{u}_{s}+f_{n}\left(\bar{x}_{n}\right)-f_{n}\left(\overline{\hat{x}}_{n s}\right)+\varepsilon_{n}(t),
\end{aligned}
$$

where

$$
\begin{aligned}
\varepsilon_{1}(t)= & f_{1}\left(\hat{x}_{1 s}\right)-f_{1}\left(x_{1 s}\right)+\hat{x}_{2 s}-x_{2 s}, \\
\varepsilon_{i}(t)= & f_{i}\left(\overline{\hat{x}}_{i s}\right)-f_{i}\left(\bar{x}_{i s}\right)+\hat{x}_{(i+1) s} \\
& -x_{(i+1) s}+\dot{x}_{i s}-\hat{\dot{x}}_{i s},
\end{aligned}
$$

for $i \in \mathbb{N}_{2: n}$. To proceed the proof, the following proposition is required.

Proposition 1. With the help of Lemmas 1 and 2 , there is the following inequality:

$$
\begin{aligned}
\dot{V}_{n}\left(\bar{\eta}_{n}\right) \leq & \left(\tilde{c}_{n-1}\left(\bar{\eta}_{n-1}\right)+\hat{c}_{n}\left(\bar{\eta}_{n}\right)+c_{n}\right)\left\lfloor\xi_{n}\right\rceil^{\frac{2 \mu}{\rho}} \\
& +\frac{1}{2} \sum_{j=1}^{n-1}\left\lfloor\varepsilon_{j}\right\rceil^{\frac{2 \mu}{r_{j+1}}}-\sum_{j=1}^{n-1}\left\lfloor\xi_{j}\right\rceil^{\frac{2 \mu}{\rho}} \\
& +\left\lfloor\xi_{n}\right\rceil^{\frac{2 \mu-r_{n+1}}{\rho}}\left(u-\hat{u}_{s}+f_{n}\left(\bar{x}_{n}\right)\right. \\
& \left.-f_{n}\left(\overline{\hat{x}}_{n s}\right)+\varepsilon_{n}\right),
\end{aligned}
$$

with $\tilde{c}_{n-1}\left(\bar{\eta}_{n-1}\right)$ and $\hat{c}_{n}\left(\bar{\eta}_{n}\right)$ are nonlinear functions with respect to $\bar{\eta}_{n-1}$ and $\bar{\eta}_{n}$, respectively, and $c_{n}$ is a constant.

The detailed proof for Proposition 1 can be referred to Appendix A.

Define $v(t)=\varepsilon_{n}(t)-k_{2} \int_{0}^{t}\lfloor s(\tau)\rceil^{0} d \tau$. Taking the derivatives of $s$ and $v(t)$ along system dynamics, one obtains

$$
\begin{aligned}
& \dot{s}(t)=-k_{1}\lfloor s(t)\rceil^{\frac{1}{2}}+v(t), \\
& \dot{v}(t)=-k_{2}\lfloor s(t)\rceil^{0}+o(t),
\end{aligned}
$$


where $o(t)=\dot{\varepsilon}_{n}$. It is supposed that there exists a positive constant $\delta$ such that $o(t) \leq \delta$. Define a quadratic Lyapunov function $V_{s}(s, v)=\zeta^{T} P \zeta$ with $\zeta=\left[\left\lfloor\left. s(t)\right|^{\frac{1}{2}}, v(t)\right]^{T}\right.$ and $P=\left[\begin{array}{cc}4 k_{2}+k_{1}^{2} & -k_{1} \\ -k_{1} & 2\end{array}\right]$. The derivative of $V_{s}(s, v)$ is given as follows

$$
\begin{aligned}
\dot{V}(s, v) & =\frac{1}{|s|^{\frac{1}{2}}} \zeta^{T}\left(A^{T} P+A P\right) \zeta \\
& \leq-\frac{1}{|s|^{\frac{1}{2}}} \zeta^{T} Q \zeta \leq-\gamma_{s} V_{s}^{\frac{1}{2}},
\end{aligned}
$$

where $Q=\frac{k_{1}}{2}\left[\begin{array}{cc}4 k_{2}+2 k_{1}^{2}+4 \delta & -2 k_{1}-\frac{4 \delta}{k_{1}} \\ -2 k_{1}-\frac{4 \delta}{k_{1}} & 2\end{array}\right], \gamma_{s}=$ $\frac{\lambda_{\min }^{\frac{1}{2}}\{P\} \lambda_{\min }\{Q\}}{\lambda_{\max }\{P\}}$, with $\lambda_{\min }\{Q\}$ is the minimum eigenvalue of matrix $Q, \lambda_{\max }\{P\}$ and $\lambda_{\min }\{P\}$ are maximum and minimum eigenvalues of $P$, respectively.

It follows from [33] and (19) that the system states will reach the sliding manifold in a finite time, i.e. there exists a finite time $T_{r}=$ $\frac{2 V_{s}^{\frac{1}{2}}(s(0), v(0))}{\gamma_{s}}$ such that $V_{s}(s(t), v(t))=0, s(t)=0$ and $v(t)=0$ for $t \geq T_{r}$. It can be further obtained from (18) that $\dot{s}=0$ for $t \geq T_{r}$.

According to Bihari's inequality, it obtains

$$
\begin{aligned}
\|\zeta\| & \leq V_{s}^{\frac{1}{2}} / \lambda_{\min }\{P\} \leq V_{s}^{\frac{1}{2}}(0) / \lambda_{\min }\{P\} \\
& =\zeta_{\max }
\end{aligned}
$$

It can be derived from (20) that

$$
\begin{aligned}
& \left|\varepsilon_{n}-k_{1}\lfloor s\rceil^{\frac{1}{2}}-k_{2} \int_{0}^{t}\lfloor s\rceil^{0} d \tau\right|=\left|v-k_{1}\lfloor s\rceil^{\frac{1}{2}}\right| \\
& \leq\|\zeta\| \sqrt{2 \max \left\{k_{1}^{2}, 1\right\}} \leq \zeta_{\max } \sqrt{2 \max \left\{k_{1}^{2}, 1\right\}} .
\end{aligned}
$$

By Lemma 2 and inequality (21), one has

$$
\begin{aligned}
& \left\lfloor\xi_{n}\right\rceil^{\frac{2 \mu-r_{n+1}}{\rho}}\left(\varepsilon_{n}-k_{1}\lfloor s\rceil^{\frac{1}{2}}-k_{2} \int_{0}^{t}\lfloor s\rceil^{0} d \tau\right) \\
& \leq \check{c}\left|\xi_{n}\right|^{\frac{2 \mu}{\rho}}+m,
\end{aligned}
$$

where $\check{c}$ is a positive constant that can be arbi- trarily small, and

$$
\begin{aligned}
m= & \frac{r_{n+1}}{2 \mu}\left(\frac{2 \mu-r_{n+1}}{2 \mu \check{c}}\right)^{\frac{2 \mu\left(2 \mu-r_{n+1}\right)}{\rho r_{n+1}}} \\
& \times\left(\zeta_{\max } \sqrt{2 \max \left\{k_{1}^{2}, 1\right\}}\right)^{\frac{2 \mu}{r_{n+1}}} .
\end{aligned}
$$

Substituting (9)-(12) and (22) into (17) obtains

$$
\dot{V}_{n}\left(\bar{\eta}_{n}\right) \leq-\sum_{j=1}^{n}\left|\xi_{j}\right|^{\frac{2 \mu}{\rho}}+\sum_{j=1}^{n-1}\left|\varepsilon_{j}\right|^{\frac{2 \mu}{r_{j}+1}}+m
$$

where the control gain $\lambda_{n}\left(\bar{\eta}_{n}\right) \geq-1+\tilde{c}_{n-1}\left(\bar{\eta}_{n-1}\right)+$ $\hat{c}_{n}\left(\bar{\eta}_{n}\right)+\bar{c}_{n}+\check{c}+m$.

Since $f_{i} \in \mathcal{C}^{0}$, it directly follows from Lemma 4 and (16) that $\varepsilon_{i} \in \mathcal{L}_{\infty}$ and $\varepsilon_{i}=0$ for $t>T_{2}$. Therefore, there exists a constant $\beta>0$ such that $\beta=\sup _{t>0}\left(\sum_{j=1}^{n-1}\left\lfloor\varepsilon_{j}\right\rceil^{\frac{2 \rho}{r_{j+1}}}(t)\right)$. From the analysis above, we can conclude that the closed-loop system will not escape to infinity during the transient of FTDO. Since $\varepsilon_{i} \in \mathcal{L}_{\infty}, \eta_{i} \in \mathcal{L}_{\infty}$ and $\varepsilon_{i}(t)=0$ for $t \geq T_{2}$, we have

$$
\dot{V}_{n}\left(\bar{\eta}_{n}\right) \leq-\sum_{j=1}^{n}\left|\xi_{j}\right|^{\frac{2 \mu}{\rho}}+m,
$$

for all $t \geq T_{2}$. To proceed the stability analysis, we have the following proposition whose proof is included in Appendix.

Proposition 2. The closed-loop system states will not escape into infinity in a finite time and can be bounded by

$$
\left|\eta_{i}\right| \leq \delta_{i}\left(\bar{\eta}_{n}(0), \tau, \bar{r}\right)
$$

where $\delta_{i}\left(\bar{\eta}_{n}(0), \tau, \bar{r}\right)$ is a constant in terms of initial states and controller parameters.

Since $\dot{s}=0$ for all $t \geq T_{r}$, according to (18) we obtain $\varepsilon_{n}-k_{1}\lfloor s\rceil^{\frac{1}{2}}-k_{2} \int_{0}^{t}\lfloor s\rceil^{0} d \tau=0$ for $t \geq T_{r}$. Based on Proposition 2, we have $\dot{V}_{n}\left(\bar{\eta}_{n}\right) \leq-\sum_{j=1}^{n}\left|\xi_{j}\right|^{\frac{2 \mu}{\rho}}$. According to LaSalle invariant principal, $\xi_{i}(t) \rightarrow 0, \eta_{i}(t) \rightarrow 0$ and hence 
$x_{1}(t) \rightarrow r(t)$ as $t \rightarrow \infty$, which shows the asymptotically exact tracking performance of the closedloop system.

By definition of $V_{n}$ and Lemma 1 , we have the following inequality

$$
V_{n}\left(\bar{\eta}_{n}\right) \leq \vartheta \sum_{j=1}^{n}\left|\xi_{i}\right|^{\frac{2 \mu-\tau}{\rho}},
$$

where $\vartheta$ is a positive constant. Let $\alpha=\frac{1}{2} \vartheta^{\frac{2 \mu-\tau}{2 \mu}}$, it can be induced from (24) and (26) that

$$
\dot{V}_{n}\left(\bar{\eta}_{n}\right)+\alpha V_{n}^{\frac{2 \mu}{2 \mu-\tau}}\left(\bar{\eta}_{n}\right) \leq-\frac{1}{2} \sum_{j=1}^{n}\left|\xi_{n}\right|^{\frac{2 \mu}{\rho}},
$$

for $t \geq \max \left\{T_{r}, T_{2}\right\}$. By Lemma 3, it follows from $(27)$ that $\xi_{i}(t) \rightarrow 0, \eta_{i}(t) \rightarrow 0$ and subsequently $x_{i}(t) \rightarrow x_{i s}(t)$ as $t \rightarrow T$, where $\epsilon=$ $\left(r_{1} / \rho-1\right)^{2 \mu /(2 \mu-\tau)}, T=\max \left\{T_{r}, T_{2}\right\}+2^{n-1}(2 \mu-$ $\tau) V_{n}^{\frac{-\tau}{2 \mu-\tau}}(\vec{\eta}(0)) /(-\epsilon \tau)$.

The block diagram of the proposed continuous nonsingular terminal sliding mode control scheme is shown in Fig. 1.

Remark 2 It can be seen that there are some parameters in the controller to be assigned. In the finite-time disturbance observer, the parameter $L_{i}$ should be larger than the upper bounds of disturbances $\omega_{i}$ and their derivatives to ensure that the estimation errors converge to zero in a finite-time. According to [2], a possible choice of the differentiator parameters $\lambda_{j}^{i}$ for $n \leq 5$ is $\lambda_{0}^{i}=1.1$, $\lambda_{1}^{i}=1.5, \lambda_{2}^{i}=3, \lambda_{3}^{i}=5, \lambda_{4}^{i}=8, \lambda_{5}^{i}=12 . A n-$ other possible choice is $\lambda_{0}^{i}=1.1, \lambda_{1}^{i}=1.5, \lambda_{2}^{i}=2$, $\lambda_{3}^{i}=3, \lambda_{4}^{i}=5, \lambda_{5}^{i}=8$. For other cases, the details are provided in [2] and [26]. The feedback control gains $k_{1}$ and $k_{2}$ should be positive. Large values of feedback control gains will reduce the settling time but the overshoot and the control efforts will be large. Generally, when choosing the parameters, one needs to balance the overshoot, the settling time, and the control efforts.

\section{Simulations}

In this section, a single-link manipulator system [34] including actuator dynamics is considered. The system mathematical model is given by

$$
\begin{aligned}
M \ddot{q}+B \dot{q}+N \sin (q) & =I+d_{2}, \\
D \dot{I}+H I & =V-K_{m} \dot{q}+d_{3},
\end{aligned}
$$

where $q, \dot{q}$ and $\ddot{q}$ denote the link angular position, velocity and acceleration, respectively, $I$ is the motor current, $V$ denotes the voltage to be used in the control input, $M$ denotes the lumped inertia, $B$ is the friction coefficient, $N$ denotes the lumped load term, $D$ is the rotor inductance, $H$ is the rotor resistance, $K_{m}$ is the back-emf coefficient. $d_{2}$ and $d_{3}$ are unknown external disturbances. In simulation, the parameters are $M=1 \mathrm{kgm}^{2} / \mathrm{rad}$, $B=1, N=10 \mathrm{kgA}, D=0.05 \mathrm{H}, H=0.5 \Omega$ and $K_{m}=10 \mathrm{Nm} / \mathrm{A}$. Let $x_{1}=q, x_{2}=\dot{q}, x_{3}=I$ and $u=V$. The system model is written as

$$
\begin{aligned}
& \dot{x}_{1}=x_{2}, \dot{x}_{2}=x_{3}+f_{2}\left(x_{1}, x_{2}\right)+d_{2}, \\
& \dot{x}_{3}=\frac{u}{0.05}+f_{3}\left(x_{1}, x_{2}, x_{3}\right)+\bar{d}_{3}, y=x_{1},
\end{aligned}
$$

where $f_{2}\left(x_{1}, x_{2}\right)=-10 \sin \left(x_{1}\right)-x_{2}, f_{3}\left(x_{1}, x_{2}, x_{3}\right)=$ $\left(-10 x_{2}-0.5 x_{3}\right) / 0.05, \bar{d}_{3}=d_{3} / 0.05$ and $y$ denotes the output of the manipulator system.

The initial conditions are $\left[x_{1}(0), x_{2}(0), x_{3}(0)\right]^{T}=$ $[0.2,0.2,0.1]^{T}$. The reference signal to be tracked is $y_{r}=0.5 \sin (0.5 t)$, and $d_{2}=10 \sin (\pi t)$ for $t \leq$ $16 s, d_{2}=10 \sin \left(\frac{\pi}{2} t\right)$ for $t>16 s, \bar{d}_{3}=-20 \sin (\pi t)$ for $t \leq 12 s, \bar{d}_{3}=-20 \sin \left(\frac{\pi}{3} t\right)$ for $t>12 s$.

For system (29), FTDO are designed based on (3). The steady-states of $x_{i}(i=1,2,3)$ and the 


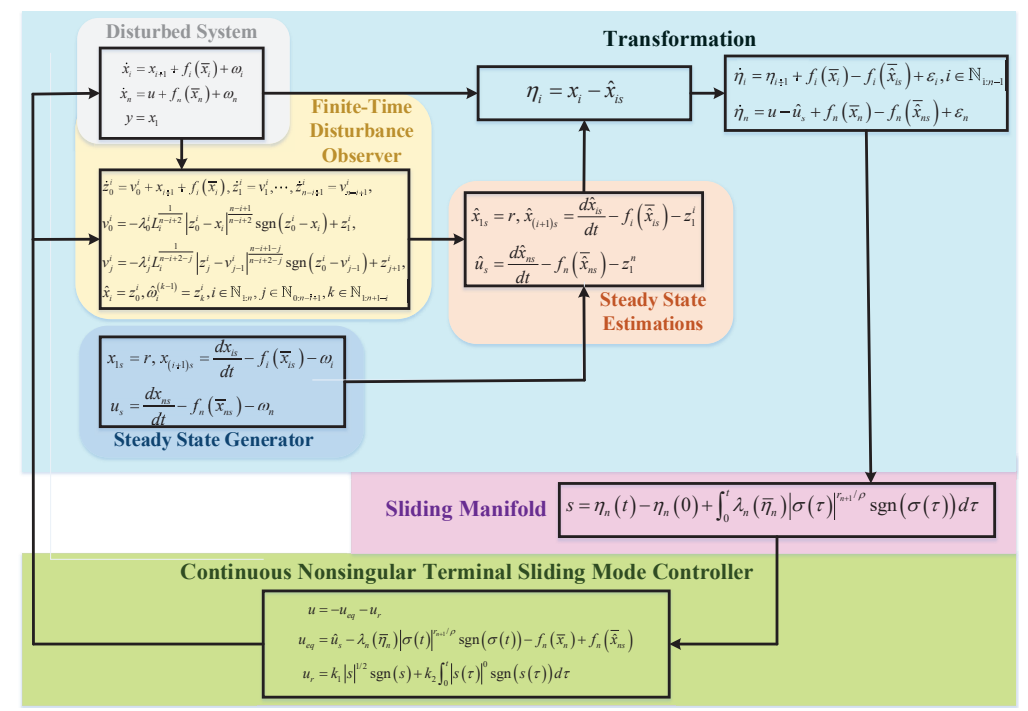

Figure 1: Implementation block diagram of the proposed continuous NTSM controller.

control input are shown as follows:

$$
\begin{aligned}
x_{1 s}= & y_{r}, \hat{x}_{2 s}=\dot{y}_{r}, \\
\hat{x}_{3 s}= & \ddot{y}_{r}+10 \sin \left(y_{r}\right)+\dot{y}_{r}-z_{1}^{2}, \\
\hat{u}_{s}= & y_{r}^{(3)}+\ddot{y}_{r}+10 \dot{y}_{r} \cos \left(y_{r}\right) \\
& +\frac{10 \hat{x}_{2 s}+0.5 \hat{x}_{3 s}}{0.05}-z_{2}^{2}-z_{1}^{3} .
\end{aligned}
$$

Denote $\eta_{1}=x_{1}-x_{1 s}, \eta_{2}=x_{2}-\hat{x}_{2 s}, \eta_{3}=x_{3}-\hat{x}_{3 s}$. The sliding manifold is designed as

$$
s=\eta_{3}(t)-\eta_{3}(0)+\int_{0}^{t} \lambda_{3}\left(\eta_{1}, \eta_{2}, \eta_{3}\right)\lfloor\sigma(\tau)\rceil^{\frac{r_{4}}{\rho}} d \tau,
$$

with $\sigma(t)=\lambda_{1}\left\lfloor\eta_{1}\right\rceil^{\frac{\rho}{r_{1}}}+\lambda_{2}\left\lfloor\eta_{2}\right\rceil^{\frac{\rho}{r_{2}}}+\lambda_{3}\left\lfloor\eta_{3}\right\rceil^{\frac{\rho}{r_{3}}}$. The NTSM controller is

$$
\begin{aligned}
u= & -0.05\left[k_{1}\lfloor s\rceil^{\frac{1}{2}}+k_{2} \int_{0}^{t}\lfloor s\rceil^{0} d \tau\right. \\
& -\lambda_{3}\left(\eta_{1}, \eta_{2}, \eta_{3}\right)\lfloor\sigma\rceil^{\frac{r_{4}}{\rho}}+\frac{10 x_{2}+0.5 x_{3}}{0.05} \\
& \left.-\frac{10 \hat{x}_{2 s}+0.5 \hat{x}_{3 s}}{0.05}+20 \hat{u}_{s}\right],
\end{aligned}
$$

where $\rho=1, r_{1}=1, \tau=-0.1, k_{1}=5, k_{2}=10$, $\lambda_{1}=10, \lambda_{2}=10, \lambda_{3}=100$.

Figs. 2 and 3 show the simulation results of the system states and the control input. It can be observed from Fig. 2 that the angular position $x_{1}$ tracks the reference signal $y_{r}$ well under the proposed NTSM controller in the presence of unknown time-varying external disturbances. Moreover, the angular velocity $x_{2}$, the motor current $x_{3}$ and the control signal $u$ reach their pre-calculated steady-states $x_{2 s}, x_{3 s}$ and $u_{s}$ after a transient period, respectively. Fig. 3 clearly shows that the steady-state estimations converge to their true values in a finite-time.

\section{Conclusion}

In this paper, a continuous NTSM control strategy is proposed to solve the problem of the finitetime exact tracking and disturbance rejection for nonlinear systems with matched and mismatched nonlinearities as well as matched and mismatched time-varying disturbances. The mismatched disturbances are exactly estimated by the disturbance observer and compensated in the designed nonlinear recursive sliding manifold. The Lyapunov theory is used to demonstrate and validate that the tracking error of the closed-loop sys- 
(a)
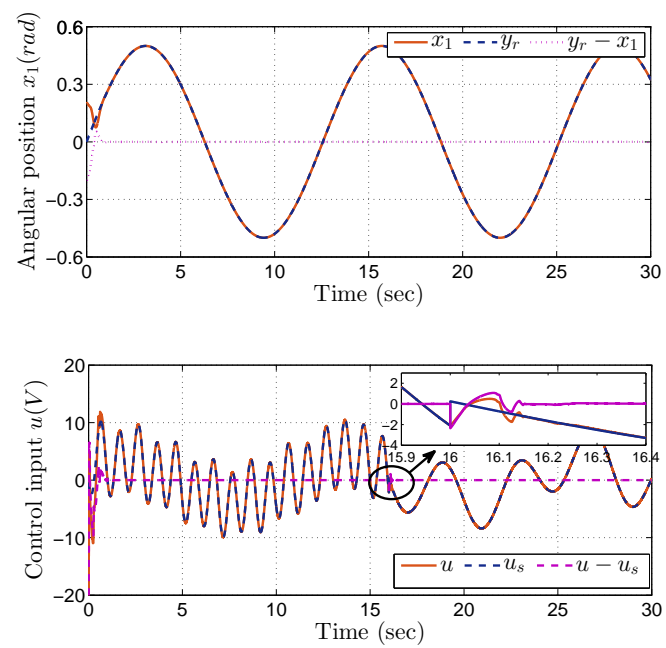

(b)

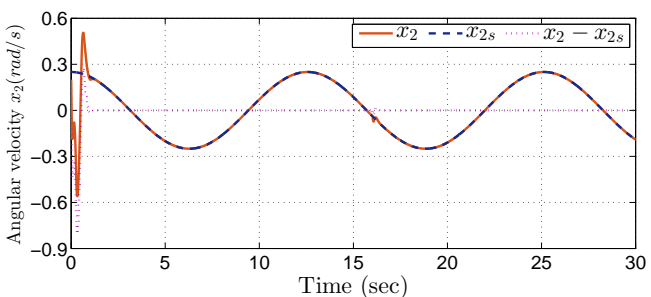

(c)

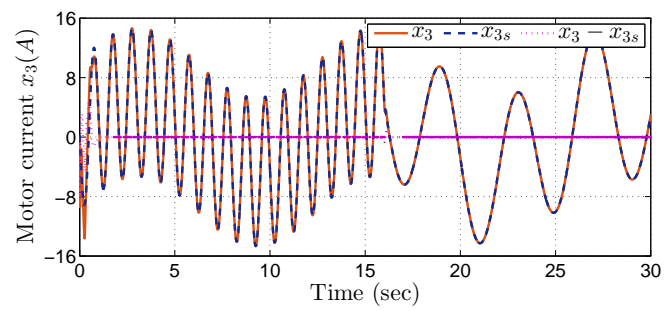

(d)

Figure 2: Response curves of system states and control signal: (a) system output $x_{1}$; (b) Control signal $u$; (c) Angular velocity $x_{2}$; (d) Motor current $x_{3}$. (a)
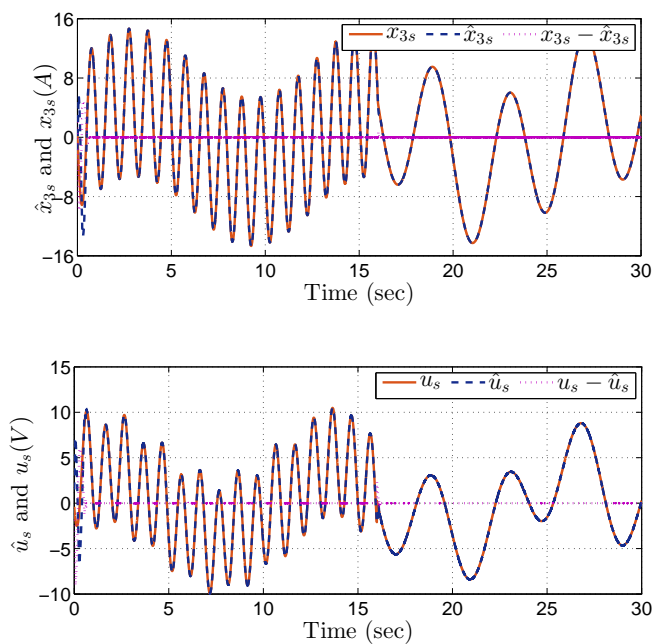

(b)

Figure 3: Response curves of estimations of the invariant variables: (a) $x_{3 s}$ and $\hat{x}_{3 s}$; (b) $u_{s}$ and $\hat{u}_{s}$.

tem converges to zero in a finite-time under the proposed controller. The simulation results verify the effectiveness of the proposed continuous NTSM controller. The finite-time sliding mode controller for multi-input-multi-output systems with unknown mismatched nonlinearities and mismatched disturbances will be considered in the future.

\section{Appendix A: proof of the Propo- sition 1}

Step 1. With the definition of $V_{i}$, one obtains

$$
V_{1}\left(\eta_{1}\right)=\int_{\eta_{1}^{*}}^{\eta_{1}}\left\lfloor\lfloor q\rceil^{\frac{\rho}{r_{1}}}-\left\lfloor\eta_{1}^{*}\right\rceil^{\frac{\rho}{r_{1}}}\right\rceil^{\frac{2 \mu-r_{2}}{\rho}} d q,
$$


By Lemma 2 and (15), the time derivative of sult, the time derivative of $V_{i}\left(\bar{\eta}_{i}\right)$ along (15) is $V_{1}\left(\eta_{1}\right)$ is

$$
\begin{aligned}
\dot{V}_{1}\left(\eta_{1}\right)= & \left\lfloor\eta_{1}\right\rceil^{\frac{2 \mu-r_{2}}{r_{1}}}\left[\eta_{2}+f_{1}\left(x_{1}\right)-f_{1}\left(\hat{\pi}_{1}\right)+\varepsilon_{1}(t)\right] \\
\leq & \left\lfloor\eta_{1}\right\rceil^{\frac{2 \mu-r_{2}}{r_{1}}}\left(\eta_{2}-\eta_{2}^{*}+\eta_{2}^{*}\right)+\frac{1}{\nu_{1}}\left\lfloor\varepsilon_{1}\right\rceil^{\frac{2 \mu}{r_{2}}} \\
& +\left[\gamma_{1}\left(\eta_{1}\right)+\varrho_{1}\right]\left\lfloor\eta_{1}\right\rceil^{\frac{2 \mu}{r_{1}}}
\end{aligned}
$$

where $\nu_{1}=n, \varrho_{1}=\frac{2 \mu-r_{2}}{2 \mu}\left(\frac{2 \mu}{r_{2} \nu_{1}}\right)^{-\frac{2 \mu r_{2}}{r_{1}\left(2 \mu-r_{2}\right)}}>0$ is a constant. By (2), it can be induced that the following inequality holds

$$
\left|f_{i}\left(\bar{x}_{i}\right)-f_{i}\left(\hat{\bar{x}}_{i s}\right)\right| \leq \gamma_{i}\left(\bar{\eta}_{i}\right) \sum_{j=1}^{i}\left|\eta_{j}\right|^{\frac{r_{i+1}}{r_{j}}},
$$

with $i \in \mathbb{N}_{1: n}, \gamma_{i}\left(\bar{\eta}_{i}\right)>0$ is a $\mathcal{C}^{0}$ function.

The virtual control law $\eta_{2}^{*}$ is designed as $\eta_{2}^{*}=$ $-k_{1}\left(\eta_{1}\right)\left\lfloor\xi_{1}\right\rceil^{\frac{r_{2}}{\rho}}$, with $\xi_{1}=\left\lfloor\eta_{1}\right\rceil^{\frac{\rho}{r_{1}}}$ and $k_{1}\left(\eta_{1}\right)$ is the control gain assigned such that $k_{1}\left(\eta_{1}\right) \geq \nu_{1}+$ $\gamma_{1}\left(\eta_{1}\right)+\varrho_{1}$. Substituting the virtual control law $\eta_{2}^{*}$ into (34) gets

$\dot{V}_{1}\left(\eta_{1}\right) \leq\left\lfloor\xi_{1}\right\rceil^{\frac{2 \mu-r_{2}}{\rho}}\left(\eta_{2}-\eta_{2}^{*}\right)-\nu_{1}\left\lfloor\xi_{1}\right\rceil^{\frac{2 \mu}{\rho}}+\frac{1}{\nu_{1}}\left\lfloor\varepsilon_{1}\right\rceil^{\frac{2 \mu}{r_{2}}}$.

Induction step. It is supposed that at step $i-1$, $V_{i-1}\left(\bar{\eta}_{i-1}\right): \mathbb{R}^{i-1} \rightarrow \mathbb{R}$ is a positive definite and proper $\mathcal{C}^{1}$ Lyapunov function. Choose nonlinear control gains $\kappa_{j}\left(\bar{\eta}_{j}\right)>0$ such that

$$
\begin{aligned}
\dot{V}_{i-1}\left(\bar{\eta}_{i-1}\right) \leq & \left\lfloor\xi_{i-1}\right\rceil^{\frac{2 \mu-r_{i}}{\rho}}\left(\eta_{i}-\eta_{i}^{*}\right)-\nu_{i-1} \sum_{j=1}^{i-1}\left\lfloor\xi_{j}\right\rceil^{\frac{2 \mu}{\rho}} \\
& +\frac{1}{\nu_{i-1}} \sum_{j=1}^{i-1}\left\lfloor\varepsilon_{j}\right\rceil^{\frac{2 \mu}{r_{j+1}}}
\end{aligned}
$$

where $\nu_{i}=n+1-i$.

Note that $V_{i}\left(\bar{\eta}_{i}\right)=V_{i-1}\left(\bar{\eta}_{i-1}\right)+W_{i}\left(\bar{\eta}_{i}\right)$ with $W_{i}\left(\bar{\eta}_{i}\right)=\int_{\eta_{i}^{*}}^{\eta_{i}}\left\lfloor\lfloor q\rceil^{\frac{\rho}{r_{i}}}-\left\lfloor\eta_{i}^{*}\right\rceil^{\frac{\rho}{r_{i}}}\right\rceil^{\frac{2 \mu-r_{i+1}}{\rho}} d q$. As a re-

$$
\begin{aligned}
\dot{V}_{i}\left(\bar{\eta}_{i}\right)= & \dot{V}_{i-1}\left(\bar{\eta}_{i-1}\right)+\left\lfloor\xi_{i}\right\rceil^{\frac{2 \mu-r_{i+1}}{\rho}} \dot{\eta}_{i}+\sum_{l=1}^{i-1} \frac{\partial W_{i}}{\partial \eta_{l}} \dot{\eta}_{l}, \\
\leq & \dot{V}_{i-1}\left(\bar{\eta}_{i-1}\right)+\left\lfloor\xi_{i}\right\rceil^{\frac{2 \mu-r_{i+1}}{\rho}}\left(\eta_{i+1}-\eta_{i+1}^{*}\right) \\
& +\sum_{l=1}^{i-1} \frac{\partial W_{i}}{\partial \eta_{l}} \dot{\eta}_{l}+\left\lfloor\xi_{i}\right\rceil^{\frac{2 \mu-r_{i+1}}{\rho}}\left(\eta_{i+1}^{*}\right. \\
& \left.+f_{i}\left(\bar{x}_{i}\right)-f_{i}\left(\hat{\bar{\pi}}_{i}\right)+\varepsilon_{i}(t)\right),
\end{aligned}
$$

for a virtual control law $\eta_{i+1}^{*}$ to be designed later. Next, each term in the right hand side of (38) is estimated.

Now that $\rho \geq r_{1}$ and $r_{i+1}=r_{i}+\tau$. By Lemma 1 and Lemma 2 , there is

$$
\left|\left\lfloor\xi_{i-1}\right\rceil^{\frac{2 \mu-r_{i}}{\rho}}\left(\eta_{i}-\eta_{i}^{*}\right)\right| \leq \frac{1}{3}\left\lfloor\xi_{i-1}\right\rceil^{\frac{2 \mu}{\rho}}+\bar{c}_{i}\left\lfloor\xi_{i}\right\rceil^{\frac{2 \mu}{\rho}},
$$

where $\bar{c}_{i}$ is a positive constant. Define $\varsigma_{l}\left(\vec{\eta}_{l}\right)=$ $\kappa_{l}^{\frac{\rho}{r_{l+1}}}\left(\vec{\eta}_{l}\right)$ for $l \in \mathbb{N}_{1: i-1}$. By Lemma 1 and (35), one obtains [36]

$$
\begin{aligned}
& \left|\left\lfloor\xi_{i}\right\rceil^{\frac{2 \mu-r_{i+1}}{\rho}}\left(f_{i}\left(\bar{x}_{i}\right)-f_{i}\left(\hat{\bar{\pi}}_{i}\right)\right)\right| \\
& \leq\left|\left\lfloor\xi_{i}\right\rceil^{\frac{2 \mu-r_{i+1}}{\rho}}\right|\left(\gamma_{i}\left(\bar{\eta}_{i}\right) \sum_{j=1}^{i}\left|\xi_{i}-\varsigma_{j-1} \xi_{i}\right|^{\frac{r_{i+1}}{\rho}}+\left|\varepsilon_{i}\right|\right) \\
& \leq \frac{1}{2} \sum_{j=1}^{i-2}\left\lfloor\xi_{j}\right\rceil^{\frac{2 \mu}{\rho}}+\frac{1}{3}\left\lfloor\xi_{i-1}\right\rceil^{\frac{2 \mu}{\rho}}+\frac{1}{\nu_{i}}\left\lfloor\varepsilon_{i}\right\rceil^{\frac{2 \mu}{r_{i+1}}} \\
& \quad+\hat{c}_{i}\left(\bar{\eta}_{i}\right)\left\lfloor\xi_{i}\right\rceil^{\frac{2 \mu}{\rho}} .
\end{aligned}
$$

By Lemma 1, Lemma 2 and (35), the following inequality can be obtained:

$$
\begin{aligned}
\sum_{l=1}^{i-1}\left|\frac{\partial W_{i}}{\partial \eta_{l}} \dot{\eta}_{l}\right| \leq & \frac{1}{2} \sum_{j=1}^{i-2}\left\lfloor\xi_{j}\right\rceil^{\frac{2 \mu}{\rho}}+\tilde{c}_{i-1}\left(\bar{\eta}_{i-1}\right)\left\lfloor\xi_{i}\right\rceil^{\frac{2 \mu}{\rho}} \\
& +\frac{1}{3}\left\lfloor\xi_{i-1}\right\rceil^{\frac{2 \mu}{\rho}}+\frac{1}{\nu_{i} \nu_{i-1}} \sum_{j=1}^{i-1}\left\lfloor\varepsilon_{j}\right\rceil^{\frac{2 \mu}{r_{j+1}}}
\end{aligned}
$$

Substituting (37) and estimations (39)-(41) in- 
to $(38)$ gets

$$
\begin{aligned}
\dot{V}_{i}\left(\bar{\eta}_{i}\right) \leq & \left\lfloor\xi_{i}\right\rceil^{\frac{2 \mu-r_{i+1}}{\rho}}\left(\eta_{i+1}-\eta_{i+1}^{*}\right)-\nu_{i} \sum_{j=1}^{i-1}\left\lfloor\xi_{j}\right\rceil^{\frac{2 \mu}{\rho}} \\
& +\left(\tilde{c}_{i-1}\left(\bar{\eta}_{i-1}\right)+\hat{c}_{i}\left(\bar{\eta}_{i}\right)+\bar{c}_{i}\right)\left\lfloor\xi_{i}\right\rceil^{\frac{2 \mu}{\rho}} \\
& +\frac{1}{\nu_{i}} \sum_{j=1}^{i}\left\lfloor\varepsilon_{j}\right\rceil^{\frac{2 \mu}{r_{j+1}}}+\left\lfloor\xi_{i}\right\rceil^{\frac{2 \mu-r_{i+1}}{\rho}} \eta_{i+1}^{*} .
\end{aligned}
$$

Substituting a virtual control law

$$
\eta_{i+1}^{*}=-k_{i}\left(\bar{\eta}_{i}\right)\left\lfloor\xi_{i}\right\rceil^{\frac{r_{i+1}}{\rho}}
$$

with $k_{i}\left(\vec{\eta}_{i}\right) \geq \nu_{i}+\tilde{c}_{i-1}\left(\vec{\eta}_{i-1}\right)+\vec{c}_{i}\left(\hat{\eta}_{i}\right)+\bar{c}_{i}$ into $(42)$ yields

$$
\begin{aligned}
\dot{V}_{i}\left(\bar{\eta}_{i}\right) \leq\left\lfloor\xi_{i}\right\rceil^{\frac{2 \mu-r_{i+1}}{\rho}}\left(\eta_{i+1}-\eta_{i+1}^{*}\right)-\nu_{i} \sum_{j=1}^{i}\left\lfloor\xi_{j}\right\rceil^{\frac{2 \mu}{\rho}} \\
+\frac{1}{\nu_{i}} \sum_{j=1}^{i}\left\lfloor\varepsilon_{j}\right\rceil^{\frac{2 \mu}{r_{j+1}}}
\end{aligned}
$$

with $i=1,2, \cdots, n-1$. This completes the induction step.

With the definition of $V_{i}$ in mind, combining (15) and (43), the following inequality is obtained

$$
\begin{aligned}
\dot{V}_{n}\left(\bar{\eta}_{n}\right) \leq & \left(\tilde{c}_{n-1}\left(\bar{\eta}_{n-1}\right)+\hat{c}_{n}\left(\bar{\eta}_{n}\right)+\bar{c}_{n}\right)\left\lfloor\xi_{n}\right\rceil^{\frac{2 \mu}{\rho}} \\
& +\frac{1}{2} \sum_{j=1}^{n-1}\left\lfloor\varepsilon_{j}\right\rceil^{\frac{2 \mu}{r_{j+1}}}-\nu_{n} \sum_{j=1}^{n-1}\left\lfloor\xi_{j}\right\rceil^{\frac{2 \mu}{\rho}} \\
& +\left\lfloor\xi_{n}\right\rceil^{\frac{2 \mu-r_{n+1}}{\rho}}\left(u-\hat{\pi}_{u}+f_{n}\left(\bar{x}_{n}\right)\right. \\
& \left.-f_{n}\left(\hat{\bar{\pi}}_{n}\right)+\varepsilon_{n}\right) .
\end{aligned}
$$

This completes the proof.

\section{Appendix B: proof of proposition}

\section{2}

Define a composite Lyapunov function as follows:

$$
V\left(\bar{\eta}_{n}, s, v\right)=V_{n}\left(\bar{\eta}_{n}\right)+\widetilde{V}_{s}(s, v)
$$

with $\widetilde{V}_{s}(s, v)=\hbar V_{s}^{\frac{2 \mu-\tau}{-2 \tau}}(s, v)$, where

$$
\hbar=\left(\frac{2 \epsilon}{(1-2 \mu / \tau) \gamma_{s}}\right)^{\frac{2 \mu-\tau}{-\tau}}, \epsilon=\left(\frac{r_{1}}{\rho}-1\right)^{\frac{2 \mu}{2 \mu-\tau}} .
$$

Since $\tau \in(-\infty, 0)$, the function $V\left(\vec{\eta}_{n}, s, v\right)$ is positive definite. Combining (19) and (24), the derivative of $V\left(\bar{\eta}_{n}, s, v\right)$ yields

$$
\begin{aligned}
\dot{V}\left(\bar{\eta}_{n}, s, v\right) \leq & -\hbar \gamma_{s}\left(\frac{1}{2}-\frac{\mu}{\tau}\right) V_{s}^{\frac{\mu}{-\tau}}(s, v) \\
& -\sum_{i=1}^{n}\lfloor\xi\rceil^{\frac{2 \mu}{\rho}}+m .
\end{aligned}
$$

By definition of $V_{n}\left(\bar{\eta}_{n}\right)$, it follows from the properties of homogeneity that $\epsilon V_{n}^{\frac{2 \mu}{2 \mu-\tau}}\left(\bar{\eta}_{n}\right) \leq \sum_{i=1}^{n}\left\lfloor\xi_{i}\right\rceil^{\frac{2 \mu}{\rho}}$, we have

$$
\begin{aligned}
\dot{V}\left(\bar{\eta}_{n}, s, v\right) & \leq-\epsilon\left(V_{n}^{\frac{2 \mu}{2 \mu-\tau}}\left(\bar{\eta}_{n}\right)+\widetilde{V}_{s}^{\frac{2 \mu}{2 \mu-\tau}}(s, v)\right)+m, \\
& \leq-\epsilon V^{c}\left(\bar{\eta}_{n}, s, v\right)+m,
\end{aligned}
$$

where $c=\frac{2 \mu}{2 \mu-\tau} \in(0,1)$. Denote $V(t)$ as the solution of $V\left(\bar{\eta}_{n}, s, v\right)$ for neatness. By comparison lemma given in [35], the solution $V(t)$ satisfies

$V(t) \leq\left\{\begin{array}{cc}{\left[V^{1-c}(0)-\epsilon(1-c) t\right]^{\frac{1}{1-c}}+V_{u}, 0 \leq t \leq T_{u}} \\ V_{u}, & t \geq T_{u}\end{array}\right.$

where $V(0)=V\left(\bar{\eta}_{n}(0), s(0), v(0)\right)$ denotes the initial value of $V\left(\bar{\eta}_{n}, s, v\right)$, and $V_{u}=\left(\frac{m}{\epsilon}\right)^{\frac{2 \mu-\tau}{2 \mu}}, \quad T_{u}=$ $\frac{(2 \mu-\tau) V^{\frac{-\tau}{2 \mu-\tau}}(0)}{-\epsilon \tau}$ represent the ultimate bound and convergence time of $V(t)$. This implies that the system states and control input are ultimate bound, i.e., $\eta_{i} \in \mathcal{L}_{\infty}, u \in \mathcal{L}_{\infty}$ and $s \in \mathcal{L}_{\infty}$.

The homogeneous degree of $V_{n}\left(\bar{\eta}_{n}\right)$ is $\frac{2 \mu-\tau}{2}$. By homogeneity property, there exists a positive constant $\underline{c}$ such that $\underline{c}\left\|\bar{\eta}_{n}\right\|_{\Delta^{r}}^{\frac{2 \mu-\tau}{2}} \leq V_{n}\left(\bar{\eta}_{n}\right) \leq$ $V\left(\bar{\eta}_{n}, s, v\right)$. As a result, the boundness of system states is given by

$$
\left|\eta_{i}(t)\right| \leq\left\|\bar{\eta}_{n}\right\|_{\Delta^{r}}^{r_{i}} \leq \delta_{i}\left(\bar{\eta}_{n}(0), \tau, \bar{r}\right),
$$


where $\delta_{i}\left(\bar{\eta}_{n}(0), \tau, \bar{r}\right)=\left(\frac{V(0)+V_{u}}{\underline{c}}\right)^{\frac{2 r_{i}}{2 \mu-\tau}} \cdot$ This completes the proof.

\section{Conflict of interest}

The authors declare no potential conflict of interests.

\section{REFERENCE}

1 Utkin V. Sliding Modes in Control and Optimization. Berlin, Germany: Springer Verlag. 1992.

2 Shtessel Y, Edwards C, Fridman L, Levant A. Sliding Mode Control and Observation. New York, USA: Birkhauser. 2014.

3 Man Z, Yu X. Terminal sliding mode control of mimo linear systems. IEEE Trans. Circuits Syst I: Fundam Theory Appl. 1997; 44(11): 10651070.

4 Feng Y, Yu X, Man Z. Non-singular terminal sliding mode control of rigid manipulators. Automatica 2002; 38(12): 2159-2167.

5 Wang H, Shi L, Man Z, et al. Continuous fast nonsingular terminal sliding mode control of automotive electronic throttle systems using finitetime exact observer. IEEE Trans. Ind. Electron. 2018; 65(9): 7160-7172.

6 Qiao L, Zhang W. Trajectory tracking control of AUVs via adaptive fast nonsingular integral terminal sliding mode control. IEEE Trans. Ind. Informat. 2020; 16(2): 1248-1258.

7 Zhao W, Ren X, Li L. Synchronization and tracking control for dual-motor driving servo systems with friction compensation. Asian J. Control 2019; 21(2): 674-685.

8 Van M, Ge S, Ren H. Finite time fault tolerant control for robot manipulators using time delay estimation and continuous nonsingular fast terminal sliding mode control. IEEE Trans. Cyber. 2017; 47(7): 1681-1693.

9 Chen W, Yang J, Guo L, Li S. Disturbance observer-based control and related methods-an overview. IEEE Trans. Ind. Electron. 2016; 63(2): 1083-1095.
10 Kayacan E, Fossen T. Feedback linearization control for systems with mismatched uncertainties via disturbance observers. Asian J. Control 2019; 21(3): 1064-1076.

11 Yan Y, Yang J, Sun Z, Zhang C, Li S, Yu H. Robust speed regulation for PMSM servo system with multiple sources of disturbances via an augmented disturbance observer. IEEE/ASME Trans. Mechatronics 2018; 23(2): 769-780.

12 Tapia A, Bernal M, Fridman L. Nonlinear sliding mode control design: an LMI approach. Syst. Control Lett. 2017; 104: 38-44.

13 Bandyopadhyay B, Deepak F, Kim KS. Sliding mode control using novel sliding surfaces. Berlin, Germany: Springer-Verlag. 2009.

14 Fang X, Liu F. High-order mismatched disturbance rejection control for small-scale unmanned helicopter via continuous nonsingular terminal sliding-mode approach. Int. J. Robust Nonlinear Control 2019; 29: 935-948.

15 Wang X, Li S, Yu X, Yang J. Distributed active anti-disturbance consensus for leader-follower higherorder multi-agent systems with mismatched disturbances. IEEE Trans. Autom. Control 2017; 62(11) : 5795-5801.

16 Wang J, Li S, Yang J, Wu B, Li Q. Finite-time disturbance observer based non-singular terminal sliding-mode control for pulse width modulation based DC-DC buck converters with mismatched load disturbances. IET Power Electron. 2016; 9(9): 1995-2002.

17 Wang J, Zong Q, Su R, Tian B. Continuous high order sliding mode controller design for a flexible air-breathing hypersonic vehicle. ISA Trans. 2014; 53: 690-698.

18 Choi H. Variable structure output feedback control design for a class of uncertain dynamic systems. Automatica 2002; 38: 335-341.

19 Tian B, Lu H, Zuo Z, Wang H. Fixed-time stabilization of high-order integrator systems with mismatched disturbances. Nonlinear Dyn. 2018; 94: 2889-2899.

20 Ding S, Zheng W. Nonsingular terminal sliding mode control of nonlinear second-order systems with input saturation. Int. J. Robust Nonlinear Control 2016; 26: 1857- 1872.

21 Feng Y, Zheng X, Yu X. Second-order nonsignular terminal sliding mode decomposed control of 
uncertain multi-variable systems. Asian J. Control 2003; 5(4): 505-512.

22 Tao C, Taur J, Chan M. Adaptive fuzzy terminal sliding mode controller for linear systems with mismatched time-varying uncertainties. IEEE Trans. Syst. Man Cybern. Syst. Part B Cybern. 2004; 34(1): 255-262.

23 Feng Y, Zhou M, Zheng X, Han F, Yu X. Fullorder terminal sliding-mode control of MIMO systems with unmatched uncertainties. J. Frankl. Inst. 2018; 355: 653-674.

24 Sun H, Li S, Sun C. Finite time integral sliding mode control of hypersonic vehicles. Nonlinear Dyn. 2013; 73: 229-244.

25 Yang J, Li S, Su J, Yu X. Continuous nonsingular terminal sliding mode control for systems with mismatched disturbances. Automatica 2013; 49: 2287-2291.

26 Levant A. Higher-order sliding mode, differentiation and output feedback control. Int. J. Control 2003; 76(9-10): 924-941.

27 Qiao J, Li Z, Xu J, Yu X. Composite nonsingular terminal sliding mode attitude controller for spacecraft with actuator dynamics under matched and mismatched disturbances. IEEE Trans. Ind. Informat. 2020; 16(2): 1153-1162.

28 Isidori A, Byrnes C. Output regulation of nonlinear systems. IEEE Trans. Autom. Control 1990; 35(2): 131-140.

29 Chen Z, Huang J. Stabilization and Regulation of Nonlinear Systems. Cham, Switzerland: Springer. 2015.

30 Chen Z, Xu D. Output regulation and active disturbance rejection control: unified formulation and comparison. Asian J. Control 2016; 18(5): 1668-1678.

31 Qian C, Lin W. A continuous feedback approach to global strong stabilization of nonlinear systems. IEEE Trans. Autom. Control 2001; 46: 1061-1079.

32 Hardy G, Littlewood J, Polya G. Inequalities. Cambridge University Press. 1952.

33 Moreno J, Osorio M. Strict Lyapunov function for the super-twisting algorithm. IEEE Trans. Autom. Control 2012; 57(4): 1035-1040.

34 Zhou J Y, Zhou R J, Wang Y Y. Robust nonlinear reduced-order dynamic controller design and its application to a single-link manipulator. in Proc. IEEE Int. Conf. Robot. Autom. 2001; 2: 1149-1154.

35 Khalil H. Nonlinear Systems. New Jersey: Prentice Hall. 2002.

36 Polendo J, Qian C, An expanded method to robustly stabilize uncertain nonlinear systems, Commun. Inform. Syst., 2008; 8(1): 55-70.

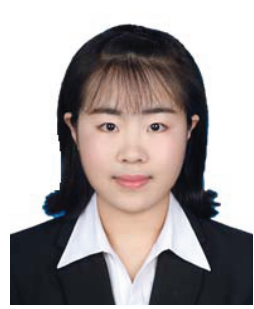

Lu Zhang received her bachelor degree in School of Control Science and Engineering from Hebei University of Technology, Tianjin, China in 2015. In 2017, she received her master degree in control theory and control engineering from School of Automation, Southeast University, Nanjing, China, where she is currently working toward Ph.D degree. Her research interests include disturbance rejection control, sliding mode control and its application to motion control systems.

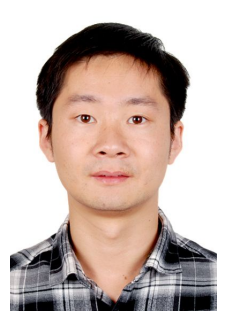

Jun Yang (SM'18) received the B.Sc. degree in automation from the Department of Automatic Control, Northeastern University, Shenyang, China, in 2006, and the Ph.D. degree in control theory and control engineering from the School of Automation, Southeast University, Nanjing, China, 2011. He joined the Department of Aeronautical and Automotive Engineering at Loughborough University from 2020 as a Senior Lecturer. His research interests include disturbance estimation and compensation, advanced control theory and its application to flight control systems and motion control systems. 